\title{
A New Vaccine for Tuberculosis: The Challenges of Development and Deployment
}

\author{
Helen A. Fletcher • Tony Hawkridge • \\ Helen McShane
}

Received: 3 March 2009 / Accepted: 24 March 2009/Published online: 5 May 2009

(C) The Author(s) 2009. This article is published with open access at Springerlink.com

\begin{abstract}
Tuberculosis (TB) is one of the world's leading causes of death due to infection and efforts to control TB would be substantially aided by the availability of an improved TB vaccine. There are currently nine new TB vaccines in clinical development, and the first efficacy trials are due to commence in 2009. There are many complex ethical issues which arise at all stages of TB vaccine development, from the need to conduct trials in developing countries to informed consent and the process of ethical review. While it is important that these issues are discussed, it may also be timely to consider the challenges which may arise if a vaccine in clinical development proves to be highly effective. We examine a number of scenarios where decisions on the deployment of a
\end{abstract}

Competing Interest Statement Helen McShane is an inventor on a composition of matter patent and a shareholder in a Joint Venture, Oxford-Emergent Tuberculosis Consortium, formed to develop MVA85A. The views expressed in this article are those of the author(s) and do not necessarily represent those of the Oxford-Emergent Tuberculosis Consortium Ltd

H. A. Fletcher $(\bowtie) \cdot$ H. McShane

The Jenner Institute, Nuffield Department of Clinical

Medicine, The University of Oxford,

Oxford, UK

e-mail: helen.fletcher@ndm.ox.ac.uk

T. Hawkridge

Aeras Global TB Vaccine Foundation, Africa Office,

Cape Town, South Africa new TB vaccine may impact on the rights and liberty of the individual.

Keywords BCG vaccine Tuberculosis vaccines . Biological markers $\cdot$ Tuberculosis

\section{Introduction}

The World Health Organization (WHO) declared tuberculosis (TB) a global health emergency in 1993 with good reason; sixteen years later the scale of the problem remains an urgent global challenge. With more than 9 million new cases and almost two million deaths each year, TB is one of the world's leading infectious causes of illness and death (WHO 2007). Effective methods of diagnosing and treating TB already exist and yet, due to (population increases and) increases in HIV-associated TB in Africa and the emergence of multidrug-resistant strains of Mycobacterium tuberculosis $(M . t b)$, the global incidence of $\mathrm{TB}$ is increasing (Corbett et al. 2003). Despite its global impact there has been a lack of discussion of the ethical issues related to TB (Selgelid et al. 2008a, 2008b). These issues include individual obligations to avoid infecting others, coercive social distancing measures to prevent the spread of infection, breaching confidentiality through notification of contacts of TB patients and health workers' duty to treat contagious patients (Selgelid et al. 2008b). There are both moral and self-interested reasons for wealthy nations to do 
more to help reduce the burden of TB in developing countries; and ethicists should evaluate whether it would be more effective to focus spending on research and development, or on improving healthcare infrastructure. Ethical policy for infectious disease control should aim to strike a balance between protecting rights/liberties of infected individuals and promoting the greater good of society in the way of public health (Selgelid 2005). Many of the ethical issues related to infectious disease, such as coercive social distancing and breaching of confidentiality through notification of contacts, could be avoided if diseases were eradicated or infection prevented. The most effective and cost-effective tool for disease prevention is vaccination. The only existing licensed vaccine against TB is a substrain of $M$. bovis, Bacille Calmette Guérin, or BCG. This vaccine, developed around the turn of the last century and first administered in 1921, is given to a large proportion of newborn infants throughout the world in the hope that it will protect them against TB. However, the protection BCG confers against TB is both incomplete and variable (Colditz et al. 1994).

We submit that a new TB vaccine, or vaccine regimen, which would ideally be effective in all populations and all age groups, against all stages of TB infection and disease (for example latent, pulmonary and extra-pulmonary TB) is the only affordable and realistic long-term solution to the global TB epidemic.

There are many important ethical issues to be considered in the development of a new vaccine for an epidemic disease such as TB. As with all medical research on humans, TB vaccine trials must be conducted according to the principals of the Declaration of Helsinki, most recently updated in 2008 (WMA 2008), and the Belmont Report (U. S. Department of Health, Education and Welfare 1979). The latter establishes the principles of 1) Respect for persons 2) Beneficence and 3) Justice. Most previously published work on TB vaccines has focused primarily on the issue of informed consent (Ibanga et al. 2006; Minnies et al. 2008; Snider 2000). Selgelid et al. have previously described the ethical issues associated with TB disease (Selgelid et al. 2008a, 2008b). Using these documents as guides, we consider the ethical challenges which face TB vaccine development and deployment; and we highlight some of the ethical issues that warrant further consideration.

\section{Challenges to Decision Making in Vaccine Development}

One of the first efficacy trials of a new generation vaccine designed to boost an antigen specific $T$ cell response was the STEP trial, which assessed the efficacy of an adenovirus type 5 (Ad5)-based vaccine designed to prevent HIV infection. In the STEP trial, vaccination of men with pre-existing Ad5 immunity unexpectedly resulted in a higher incidence of HIV-1 infection, when compared to placebo-vaccinated men (Buchbinder et al. 2008). This led to an extensive and detailed review of the preclinical efficacy and preclinical and clinical immunogenicity data obtained with the Ad5-based vaccine, to determine if the outcome of the STEP trial could have been predicted (McElrath et al. 2008). The vaccine was protective in most animal models and immunogenic in both animals and humans. This highlights two critical problems with the development of vaccines for cell mediated immunity: 1) a lack of validated preclinical models which predict the efficacy of a vaccine in humans; and 2) an incomplete understanding of the components of a protective immune response. It is important that we learn from the experiences of the STEP trial in order that we protect patient-subjects, use what limited resources we have in the most effective manner and develop and deploy a more effective TB vaccine.

\section{Progression from Pre-clinical to Clinical Testing}

One ethical issue raised by progression from preclinical to clinical vaccine testing is distributive justice. There are limited resources for conducting TB vaccine trials, so some fair means of allocating scarce resources must be determined. In addition to safety, the two primary methods used for selecting a $\mathrm{TB}$ vaccine candidate are discussed below.

\section{Efficacy Testing in Animal Models}

The first experiments which demonstrated that a subunit TB vaccine could protect mice against challenge with virulent $M$. $t b$ were published in 1996 (Huygen et al. 1996; Tascon et al. 1996). There was optimism at the time that an improved TB vaccine would soon be available for human use. Since that time, there have been hundreds of reports of experimental vaccines demonstrating protection against challenge 
with $M . t b$ in mice; yet, to date, only nine of these vaccines have progressed to clinical trials. The most commonly used murine model can be highly variable and the same vaccine candidate can demonstrate $100 \%$ protective efficacy to no protective efficacy depending on factors such as the species of animal used, the route of vaccination, the interval between vaccination and challenge, the route and dose of challenge and the interval between challenge and termination (Orme 2005). The limitations of the murine model are well illustrated by the consistent protective effect of $\mathrm{BCG}$ vaccination in mice. If $\mathrm{BCG}$ were as consistently good in humans, the need for a new TB vaccine would be much less urgent.

Limitations of the murine model have led to the experimental use of other animal species including guinea pigs, rabbits, non-human primates and cattle. All preclinical animal experiments are expensive, and time consuming (usually running for more than 6 months); and they require highly specialized Category 3 biocontainment facilities, which are not widely available.

Do we understand enough about the strengths and limitations of our models to use animal data as a basis for the selection of vaccine candidates? Is it ethical to accept or reject a candidate on the basis of preclinical animal efficacy data? It is unlikely that any candidate vaccines would progress through preclinical testing to early stage clinical testing without any evidence of efficacy. Collaborative efforts such as those funded by the NIH and European Commission Framework 6 TBVAC cluster have tested many TB vaccine candidates in multiple animal species (Baldwin et al. 1998; Williams et al. 2005). In the TBVAC project funding was provided for head-to-head testing of several new TB vaccine candidates from various sponsors. Such comparative experiments will remain necessary if we are to maximize the possibility of selecting the safest and most protective vaccine candidates for further development.

\section{Measurement of Immunogenicity}

There is no proven immunological correlate of protection with which one can select vaccines to progress from early, small scale Phase I/Ila clinical trials to late, larger scale Phase IIb/III efficacy trials.

The identification of an immunological correlate of protection or "biomarker" would greatly facilitate the development of a vaccine for TB, as such a biomarker could be used to identify protective vaccines early in clinical development. Currently, interferon-gamma (IFN- $\gamma$ ) is used as a biomarker for TB vaccine selection, despite the fact that IFN- $\gamma$ alone is insufficient for the prevention of TB disease.

Is it reasonable to select vaccine candidates on the basis of an ability to induce antigen-specific IFN- $\gamma$, when we know that this immunological measurement alone is not sufficient for protection? Although we do not yet have a better biomarker than IFN- $\gamma$, efforts should be made to harmonise the immunological assays in use for the measurement of IFN- $\gamma$ in clinical trials which are currently being conducted in various countries worldwide (Hanekom et al. 2008). Finally, it is also important that we store samples from larger efficacy trials to enable the identification of biomarkers that could be used to select promising vaccines and aid in the just distribution of the limited resources available for TB vaccine development.

Progression from Phase I Safety Trials to Phase III Efficacy Trials

Ethical principles considered here include beneficence and distributive justice.

\section{Non-inferiority, Clinical Superiority and Placebos}

A double blind, randomized, placebo controlled trial is considered the optimum design for a phase III efficacy trial. For a disease where no current vaccine is available (such as for HIV or malaria) the ethics of trial design is focused on appropriate sample size and selection of placebo. The rabies vaccine is considered a good placebo for malaria as this will offer some benefit to those participants not receiving the malaria vaccine. For a new $\mathrm{TB}$ vaccine the scenario is different due to the currently available TB vaccine, BCG. BCG is one of the most widely administered vaccines in the world and despite varying efficacy does provide some protection against childhood forms of TB. To date it has been the view of researchers, ethics committees and regulatory agencies that in a country where TB is endemic it would be unethical to withhold the $\mathrm{BCG}$ vaccine in a randomized control trial of a new TB vaccine. This view has driven the development of vaccines designed to work in combi- 
nation with BCG and slowed the development of vaccines designed to replace $B C G$. For a vaccine designed to enhance previous BCG vaccination, a randomized placebo controlled trial of the boosting vaccine is possible without withdrawing BCG vaccination. To facilitate the development of vaccines designed to replace BCG the European Commission TBVAC consortium with co-sponsorship of the Aeras Global TB Vaccine Foundation and the WHO have formed a consensus document of the proposed entry criteria for replacement vaccines (Kamath et al. 2005). The availability of the BCG vaccine also raises the question of whether a new TB vaccine should demonstrate noninferiority or clinical superiority to BCG. A noninferiority trial aims to demonstrate that the new vaccine is not worse than the comparator by more than a pre-specified, small amount. There has been much debate about the ethics of performing noninferiority trials relating to the principle of beneficence where it is the duty of healthcare workers to provide a benefit to the patient. While some argue that the introduction of a non-inferior drug would increase availability and affordability (Soliman 2008) the alternative view is that non-inferiority trials are unethical since a product tested in this way is not (afterwards) proven to offer benefit when compared to the existing treatment and may indeed be less safe and less effective than the existing treatment (Garattini and Bertele 2007). The first clinical efficacy trials of two new TB vaccines are due to commence in 2009 and both these trials are designed to show clinical superiority when compared to BCG.

\section{Lack of Global Capacity for Vaccine Efficacy Evaluation}

A major challenge facing TB vaccine developers is the limited availability of suitable field sites where TB vaccine efficacy trials can be conducted, together with a relative lack of funding for such sites. TB trials are likely to be very costly, because of their size and because of the length of follow-up required, which is directly linked to the lack of an acceptable validated immunological surrogate endpoint. Clinical efficacy endpoints take time to develop, so follow up in a TB efficacy trial is unlikely to be less than a year and probably two or more years. The result is that a restricted number of vaccine candidates can progress to Phase IIb and III efficacy trials, and efforts are being made to address this bottleneck by Aeras and by the European and Developing Countries Clinical Trials Partnership (EDCTP). Until more trial sites become available ethicists should examine how clinical trial sites are used to ensure that the principles of distributive justice are adhered to as vaccine candidates are selected for efficacy testing.

A new, more protective TB vaccine could potentially be used anywhere where BCG is currently used, including in many lower burden countries and even in areas where BCG is not currently used. The available literature on BCG shows that its efficacy in preventing TB disease varies widely with factors such as geographical latitude; for example BCG has been demonstrated to be efficacious in Britain and North America, but not so in India and Africa (Colditz et al. 1994).

Given this variability, it is possible that a new TB vaccine which is of benefit in one population may not be of benefit in different regions of the world. How many countries or regions of the world should be included in efficacy evaluations and how do you determine if such multiple evaluations are an effective use of scarce resources? Given the high burden of TB disease in sub-Saharan Africa, we feel that any new TB vaccine must first be evaluated there. It would be desirable also to show efficacy within the Indian subcontinent and in South-East Asia, in part because of the appreciable burden of disease in these two areas (both in terms of TB incidence rates, but more especially in terms of absolute numbers of cases of TB). A non-inferiority trial may be an ethical trial design when comparing the efficacy of a new TB vaccine between different countries.

\section{Exclusion of HIV Infected Individuals from Trials}

All early trials of new TB vaccines have thus far excluded HIV infected individuals. This group is considered to be at higher risk for adverse events than the HIV negative population and vaccines are only evaluated in HIV positives once safety has been demonstrated in HIV negative people. Whilst this cautious approach is logical, there is a need to evaluate new TB vaccines in the HIV-infected, since they are arguably the population most in need of a new TB vaccine, with annual TB incidence rates of between $5-10 \%$ (and potentially higher) depending on the degree of immune suppression and access to antiretroviral therapy. This higher incidence of TB 
means that efficacy can be evaluated using smaller numbers of participants, once safety (in others) has been confirmed. TB efficacy trials for HIV positive populations are currently in the planning stages and study participants will receive the usual standard of care provided by their National Control Programme. For this reason the first trials will be conducted in countries that have a National Control Programme which includes the availability of anti-retroviral drugs. To ensure safety we will work in close contact with the healthcare workers who usually provide care and HIV viral loads and CD4 counts of study participants will be closely monitored.

\section{Involvement of Pharmaceutical Companies}

Ideally, we need the involvement of pharmaceutical companies in the development of drugs and vaccines for poverty related diseases, in order to work with the Global Alliance for Vaccines and Immunization (GAVI), UNICEF and the Global Fund to ensure these products reach the markets for which they are intended in a timely fashion. Historically, these companies have been reluctant to invest time and resources into developing such products, possibly because - at least in part - of perceptions that any financial return on such an investment would be improbable. More recently, several innovative schemes and mechanisms have been established, in order to encourage pharmaceutical involvement in this field. Firstly, under the European Medicines Agency (EMEA) and the U.S. Food and Drug Administration (FDA) is the Orphan Drug Scheme (http://www.emea.europa.eu/htms/human/orphans/ intro.htm and http://www.fda.gov/orphan/). If a market is considered small enough (e.g. for a rare disease) or if the financial return on a product is likely to be very low (e.g. for prophylactic vaccination against a poverty related disease), then the developers can apply to the regulatory authorities (the FDA and/or the EMEA) to have their product designated an Orphan Drug. Such a designation provides benefit to the developer, including free regulatory advice estimated by the EMEA to be worth $€ 6,000,000$. Another benefit of being accorded Orphan Drug status is the award of 7-year and 10-year market exclusivity by the FDA and EMEA respectively. There is an ethical concern that such exclusivity could lead to excessive pricing of drugs for rare diseases (Samson 2008). As
$95 \%$ of the market for a poverty related disease, such as $\mathrm{TB}$, will be developing-country based the pricing of the vaccine will be restricted, and therefore even 10 -years market exclusivity in Europe is not expected to provide a sufficient return on investment. The exclusivity period may also be broken if the holder of the original orphan vaccine gives consent; is unable to supply sufficient quantities; or if the second vaccine is proved clinically superior.

Secondly, under the GAVI Alliance, the Advance Purchase/Market Commitment Scheme (http://www. vaccineamc.org/) involves promises by governments and agencies to procure stated quantities of new drugs and vaccines (meeting pre-specified criteria) at a predetermined price. Such commitments provide pharmaceutical companies with reassurance concerning a financial return on their investment and thus provide an incentive to conduct more research and development of drugs and vaccines for neglected diseases.

Thirdly, the FDA recently launched the Priority Review Voucher Scheme (http://www.fda.gov/cber/ gdlns/tropicaldisease.htm). In return for filing an application on a product designed for a listed disease of poverty, a company receives a voucher which allows for "priority regulatory review" of one other product that the company is developing. These vouchers may be traded and sold-and they have high expected value when used to expedite approval of potential new "blockbuster" drugs.

Such schemes make it more likely that pharmaceutical companies will invest in the development of a vaccine for a disease such as TB. Whilst they offer reasonable incentives for pharmaceutical companies, mechanisms should be established to ensure that, once licensed, such a vaccine is made available at or below (subsidized) cost-price to the governments of high TB burden countries, where it is most needed.

A related question is whether the incentives provided by the Orphan Drug Scheme are sufficient, and whether there is scope for this to increase. Just as many governments offer motorists tax breaks for using environmentally friendly cars, they might offer pharmaceutical companies tax breaks for investing in diseases such as TB, HIV and malaria. We posit that such additional incentives would increase the number of pharmaceutical companies which invest in vaccines for diseases of poverty, as well as the fraction of their 
budgets which they are prepared to commit to this end.

Registration Issues

\section{Regulatory Authority Capacity Issues}

Generally, the regulatory authorities which are the best resourced - and consequently the most capableof efficiently conducting competent and rigorous reviews of clinical development plans, clinical trial protocols and applications for licensure, are located in countries or regions of the world which do not have a high burden of TB. Examples are the FDA in the United States, the European EMEA, and the regulatory authorities of Japan and Australia. The converse is unfortunately also largely true: many high TB burden countries have poorly resourced, inexperienced regulatory authorities which do not have such capacity. In the worst case, a high burden country may have no regulatory authority and very little ethical review capacity. This potentially creates an opening for unethical, insufficiently regulated trials to be conducted by unscrupulous researchers. In the middle ground are high burden countries with some resources and some structures which can render a competent review; but, because of their resource constraints, review in such countries can take a disproportionately long time. Limited capacity and resources results in delays, not only for protocol approval but also for registration of new, effective products. In high burden countries, this is far from an ideal situation.

The WHO has established a task force with the specific aim of improving regulatory capacity in developing countries (Brennan et al. 2007). Experienced regulators from both the FDA and EMEA are working together with developing world regulators in order to build capacity, exchange experiences and provide support. Ideally, a new TB vaccine will undergo parallel registration with either the EMEA or the FDA, and simultaneously within the developing world, for example, with the South African Medicines Control Council (MCC).

One further regulatory issue relates to how wide such registration can and should be. If the efficacy of a new TB vaccine is satisfactorily demonstrated in one population in one country (e.g. in South African infants), could the vaccine, on the basis of the same results, also be registered for use in other age groups within the same country and/or for use in the same age group in other countries? Careful regulatory advice regarding bridging studies will be required.

\section{Deployment}

\section{Post Registration Issues}

When a TB vaccine is granted registration we will have an ethical obligation to ensure that it is made available to those most at risk. Given that approximately one third of the world's population is infected with $\mathrm{TB}$ and that tens of millions of infants born every year are at risk of acquiring infection, supplying enough vaccine will be a challenge and raises issues of international distributive justice.

Many public funding agencies and universities incorporate humanitarian access rights into their stated missions, statutes or charters. For example, the stated mission of the Aeras Global TB Vaccine Foundation (www.aeras.org) is to develop effective TB vaccine regimens that will prevent $T B$ in all age groups and will be affordable, available and adopted worldwide. To ensure future access, Aeras

obtains rights and/or licenses to distribute vaccines in resource-poor countries ... partners with vaccine developers that agree to adequate supply and affordable production and distribution costs ... shares financial risk to spur investment by outside vaccine developers ... [and] develops manufacturing capacity to accelerate production and distribution at the lowest possible cost.

Many other charities and funding organizations have similar aims. The mission of The Wellcome Trust (www.welcome.ac.uk) is "to foster and promote research with the aim of improving human and animal health". The European \& Developing Countries Clinical Trials Partnership (EDCTP, www.edctp.org) was created in 2003 as a European response to the global health crisis caused by the three main povertyrelated diseases of HIV/AIDS, malaria and tuberculosis. The mission of EDCTP is to accelerate the development of new or improved drugs, vaccines and microbicides against HIV/AIDS, malaria and tuberculosis, with a focus on phase II and III clinical trials in sub-Saharan Africa. To achieve this, EDCTP supports multicentre projects which combine clinical 
trials, capacity building and networking. The aim of integrating these three activities is to ensure that the developed capacity is utilised to successfully conduct the clinical trials in a sustainable way. All EDCTPfunded projects are undertaken in partnership with sub-Saharan African countries, to ensure synergy and optimal use of resources and to create a win-win situation for all parties involved.

Use of an Un-registered Vaccine

\section{Should We be Using these Vaccines as Immunotherapeutics in Drug Resistant TB?}

At the World Lung Health meeting in Paris in October 2008, early results of the "DarDar trial", a Phase III efficacy trial of a novel TB vaccine, $M$. vaccae, in HIV infected adults in Tanzania, were presented (Alcorn 2008). The trial showed a significant reduction $(37 \%)$ in the rate of culture positive TB disease in those who received the vaccine compared to those who received placebo. These results show that a TB vaccine can not only prevent disease but may be useful in the treatment of existing TB disease.

The last few years have seen the identification of extensively drug resistant strains of $M$. $t b$ (XDR TB) and a greater understanding of the extent of multi drug resistant (MDR) strains of $M . t b$ throughout the world (CDC 2006; Gandhi et al. 2006). Treatment of MDRTB and particularly XDR TB is much more expensive, more toxic and less successful than treatment of drug sensitive strains of $M . t b$

Once we have an indication that a particular new TB vaccine may be effective in preventing TB disease, should such vaccines be made available, prior to full registration and licensure for therapeutic use in patients suffering from XDR or MDR TB?

The FDA does have a mechanism for distribution of a medicinal product after efficacy has been demonstrated but before marketing has been authorized called "Treatment Investigational New Drugs" or "Treatment IND" (entered into the Federal Register, May 22, 1987). However, very few applications have been granted and safety and efficacy data on test subjects must still be collected.

It is important to consider the impact this could have on the registration process if there were an adverse event (AE). The risk of an AE if a new vaccine were to be administered to a patient with TB disease, particularly a drug-resistant strain of $M . t b$, is potentially high. It may be difficult to ascertain the causality of such an AE, and either (a) the AE may be reported as being related to vaccination, which may delay or even prevent registration of the vaccine for prophylactic use or (b) the AE may be reported as not being related to vaccination, but the timing may mean it is impossible to prove this conclusively. Development of a vaccine which may have been of enormous use as a prophylactic vaccine in one population could therefore be stopped because of the occurrence of an $\mathrm{AE}$ in very high risk group.

Further Challenges Which may Arise if a Vaccine Works

What follows are some "thought experiments" using a number of different scenarios and illustrating some of the ethical issues relating to vaccine deployment, particularly in the early stages after licensing.

\section{Justice and the Distribution of a TB Vaccine}

It is unlikely that any new vaccine will be immediately available in sufficient quantity to meet the urgent and enormous global need. In addition to vaccine availability, logistical requirements such as a public health infrastructure for vaccine deployment and cold chain storage will need to be met. This will be the case particularly if an effective vaccine is to be administered to adolescents and young adults. At present there are no routine vaccination programmes outside of infancy in most developing countries, yet adolescents and young adults form an important target population for a new TB vaccine. It is possible, therefore, that some form of prioritization will have to be instituted, at least with regard to the initial rollout of the new vaccine.

Factors which would need to be considered when prioritizing countries (or groups within countries) include the incidence (and incidence rate) of TB infection and disease; the prevalence (and prevalence rates) of HIV infection and AIDS; the ability of an immunization program to effectively deliver the new vaccine (including the ability to maintain the cold chain if required, reach all communities, maintain records and conduct post-licensing surveillance for adverse events following immunization); the presence 
of competing priorities on their health agenda (for example a health ministry may not wish to divert scarce resources from a campaign which aims to get insecticide treated bed-nets to families with young children if that is the national priority at the time); the presence or absence of political support at various levels for such a campaign (e.g. the regional offices of UNICEF and the WHO, the national health department); and the availability of other means of preventing progression from TB infection to TB disease (e.g. chemoprophylaxis). Some might argue that it would be illogical and even unethical to deploy a new vaccine unless a reasonably well-functioning TB control program is already in place; a surveillance programme, for example, would be necessary to demonstrate an effect of the vaccine on rates of TB infection and disease. However, countries or cities without such programmes will be among the places most in need of a new, effective vaccine, as decades of ineffective and suboptimal case detection and treatment programs have had little or no effect on the burden of disease there.

If efficacy trials of a vaccine demonstrate higher efficacy in some subgroups than others (e.g. one gender, a particular age group, or those with HIV infection), then it might be logical to target these groups first, if vaccine supplies are limited and if this approach is practical. Another approach would be to target the most vulnerable (e.g. those living in extreme poverty, children and the elderly) on the basis that they are less able to effectively compete for humanitarian aid and are most likely to benefit from vaccination.

\section{Duty to Treat TB Patients and Vaccination of Healthcare Workers}

We use the term "healthcare workers" here in the broadest sense and intend it to include physicians, nurses, radiographers, physiotherapists, social workers, Directly Observed Treatment, Short-course (DOTS) supporters and supervisors, support staff such as hospital cleaners: everyone with whom TB patients may come into significant contact. Healthcare workers are a special case for a number of reasons. Firstly they are at higher risk of exposure and consequently infection, because of their work. Secondly, their continued health and well being and ability to work is necessary for the welfare of those already suffering from TB and requiring or receiving
TB treatment. Thirdly, should they develop TB, they may well present a risk of infection to other patients and colleagues. Lastly, healthcare workers in some settings have been shown to have a higher prevalence of HIV infection (Connelly et al. 2007; Shisana et al. 2004), placing them more at risk of developing TB should they become infected with the bacillus.

In view of the above, it may be appropriate to consider healthcare workers as a priority group when allocation decisions regarding a new TB vaccine are made. In a developing country setting, where the prevalence of TB disease is high, this would most realistically be achieved by vaccinating all healthcare workers.

\section{Third-party Notification}

Close contacts of TB patients, particularly of patients with smear positive disease, are at increased risk of infection and $50 \%$ of those who progress on to develop disease do so within the first 1-2 years (Morrison et al. 2008). An effective new vaccine may well reduce either the establishment of infection in those exposed, or the development of disease in those infected. Prophylactic vaccination of such contacts may therefore be an effective public health measure.

It is likely that any new TB vaccine would be as effective against drug resistant strains of $M$. $t b$ as it would against drug sensitive strains. In addition, drug resistant strains of $M . t b$ are not currently considered intrinsically any more infectious than fully sensitive M. $t b s$ isolates, although patients may remain sputum smear and/or culture positive for longer (National Institute for Health and Clinical Excellence 2006). Contacts of MDR/XDR TB patients should therefore be at least as entitled to prophylactic vaccination as contacts of drug sensitive TB patients. However, the fact that therapeutic options for the treatment of drug resistant strains of $M . t b$ are more limited, less effective, more prolonged, more toxic, more expensive and less readily available in many high burden countries may mean that public health is best served by such contacts being given priority over contacts of patients with drug sensitive strains of $M$. $t b$. MDR/ XDR TB patients may voluntarily inform their close contacts of their exposure to the disease; but they sometimes choose not to do so. In the latter case an ethical dilemma arises, because a breach of patient 
confidentiality may be necessary to protect the exposed individual's right to life.

\section{Mandatory Treatment}

If there was an outbreak of XDR-TB, as for example in the Tugela Ferry outbreak (Gandhi et al. 2006), and an effective vaccine existed, a reasonable public health measure might be to "ring vaccinate" everyone in the immediate geographical area. The effectiveness of such a strategy would depend on the level of herd immunity achieved. Thus it might be necessary for a national authority to insist on vaccination, and to thereby override the rights of individuals. Such a measure may be seen as the most efficient and cost effective way to control such an outbreak.

Factors to be considered before such a decision could be made would include the magnitude of the risk (the closeness and duration of the contact, the risk of infection given contact, estimates as to the transmissibility of the strain, the risk and likely timing of disease given infection, the prognosis and treatment options and effectiveness should disease develop and the risk of adverse reactions to the vaccination) and the likely magnitude of the benefit (the demonstrated vaccine efficacy against ordinary and MDR/XDR TB).

\section{Conclusions}

There are many complex ethical issues which arise at all stages of TB vaccine development. Many of these are common to those previously described in the context of TB disease and further ethical discussion about how to strike a balance between rights/liberties of infected individuals and the greater good of society would be useful for considering the ethical issues arising from TB vaccine development. Vaccination has enabled the eradication of smallpox and virtual eradication of polio from the world. Development of a TB vaccine could be considered a moral obligation as reducing the morbidity and mortality of TB disease through the development and deployment of an effective vaccine would reduce the occurrence of conflict between the rights and liberties of the infected individual versus those of society.

Open Access This article is distributed under the terms of the Creative Commons Attribution Noncommercial License which permits any noncommercial use, distribution, and reproduction in any medium, provided the original author(s) and source are credited.

\section{References}

Alcorn, K. 2008. TB vaccine reduces new cases by almost $40 \%$ in people with HIV. Aidsmap News. 20 October. http:// www.aidsmap.com/en/news/0C1FB0AF-9BC6-4A7A8D47-7D8ABBD3C8E9.asp. Accessed 4 April 2009.

Baldwin, S.L., C. D'Souza, A.D. Roberts, B.P. Kelly, A.A. Frank, M.A. Lui, J.B. Ulmer, K. Huygen, D.M. McMurray, and I.M. Orme. 1998. Evaluation of new vaccines in the mouse and guinea pig model of tuberculosis. Infection and Immunity 66(6): 2951-2959.

Brennan, M.J., U. Fruth, J. Milstien, R. Tiernan, S. de Andrade Nishioka, and L. Chocarro. 2007. Development of new tuberculosis vaccines: A global perspective on regulatory issues. PLoS Medicine 4(8): e252. doi:10.1371/journal. pmed.0040252.

Buchbinder, S.P., D.V. Mehrotra, A. Duerr, D.W. Fitzgerald, R. Mogg, D. Li, P.B. Gilbert, J.R. Lama, M. Marmor, C. Del Rio, M.J. McElrath, D.R. Casimiro, K.M. Gottesdiener, J.A. Chodakewitz, L. Corey, and M.N. Robertson. 2008. Efficacy assessment of a cell-mediated immunity HIV-1 vaccine (the Step Study): A double-blind, randomised, placebo-controlled, test-of-concept trial. Lancet 372(9653): 1881-1893. doi:10.1016/S0140-6736(08)61591-3.

Centers for Disease Control, Prevention (CDC). 2006. Emergence of Mycobacterium tuberculosis with extensive resistance to second-line drugs-worldwide, 2000-2004. MMWR. Morbidity and Mortality Weekly Report 55(11): 301-305.

Colditz, G.A., T.F. Brewer, C.S. Berkey, M.E. Wilson, E. Burdick, H.V. Fineberg, and F. Mosteller. 1994. Efficacy of BCG vaccine in the prevention of tuberculosis. Metaanalysis of the published literature. Journal of the American Medical Association 271(9): 698-702. doi:10.1001/ jama.271.9.698.

Connelly, D., Y. Veriava, S. Roberts, J. Tsotetsi, A. Jordan, E. DeSilva, S. Rosen, and M.B. DeSilva. 2007. Prevalence of HIV infection and median CD4 counts among health care workers in South Africa. South African Medical Journal 97(2): 115-120.

Corbett, E.L., C.J. Watt, N. Walker, D. Maher, B.G. Williams, M.C. Raviglione, and C. Dye. 2003. The growing burden of tuberculosis: Global trends and interactions with the HIV epidemic. Archives of Internal Medicine 163(9): 1009-1021. doi:10.1001/archinte.163.9.1009.

Gandhi, N.R., A. Moll, A.W. Sturm, R. Pawinski, T. Govender, U. Lalloo, K. Zeller, J. Andrews, and G. Friedland. 2006. Extensively drug-resistant tuberculosis as a cause of death in patients co-infected with tuberculosis and HIV in a rural area of South Africa. Lancet 368(9547): 1575-1580. doi:10.1016/S0140-6736(06)69573-1.

Garattini, S., and V. Bertele. 2007. How can we regulate medicines better? BMJ (Clinical Research Ed.) 335(7624): 803-805. doi:10.1136/bmj.39281.615706.94. 
Hanekom, W.A., H.M. Dockrell, T.H. Ottenhoff, T.M. Doherty, H. Fletcher, H. McShane, F.F. Weichold, D.F. Hoft, S.K. Parida, and U.J. Fruth. 2008. Immunological outcomes of new tuberculosis vaccine trials: WHO panel recommendations. PLoS Medicine 5(7): e145. doi:10.1371/journal. pmed.0050145.

Huygen, K., J. Content, O. Denis, D.L. Montgomery, A.M. Yawman, R.R. Deck, C.M. DeWitt, I.M. Orme, S. Baldwin, C. D'Souza, A. Drowart, E. Lozes, P. Vandenbussche, J.P. Van Vooren, M.A. Liu, and J.B. Ulmer. 1996. Immunogenicity and protective efficacy of a tuberculosis DNA vaccine. Nature Medicine 2(8): 893-898. doi:10.1038/ nm0896-893.

Ibanga, H.B., R.H. Brookes, P.C. Hill, P.K. Owiafe, H.A. Fletcher, C. Lienhardt, A.V. Hill, R.A. Adegbola, and H. McShane. 2006. Early clinical trials with a new tuberculosis vaccine, MVA85A, in tuberculosis-endemic countries: Issues in study design. The Lancet Infectious Diseases 6(8): 522-528. doi:10.1016/S1473-3099(06)70552-7.

Kamath, A.T., U. Fruth, M.J. Brennan, R. Dobbelaer, P. Hubrechts, M.M. Ho, R.E. Mayner, J. Thole, K.B. Walker, M. Liu, and P.H. Lambert. 2005. New live mycobacterial vaccines: The Geneva consensus on essential steps towards clinical development. Vaccine 23(29): 37533761. doi:10.1016/j.vaccine.2005.03.001.

McElrath, M.J., S.C. De Rosa, Z. Moodie, S. Dubey, L. Kierstead, H. Janes, O.D. Defawe, D.K. Carter, J. Hural, R. Akondy, S.P. Buchbinder, M.N. Robertson, D.V. Mehrotra, S.G. Self, L. Corey, J.W. Shiver, and D.R. Casimiro. 2008. HIV-1 vaccine-induced immunity in the test-of-concept Step Study: A case-cohort analysis. Lancet 372(9653): 1894-1905. doi:10.1016/S0140-6736(08) 61592-5.

Minnies, D., T. Hawkridge, W. Hanekom, R. Ehrlich, L. London, and G. Hussey. 2008. Evaluation of the quality of informed consent in a vaccine field trial in a developing country setting. BMC Medical Ethics 9: 15. doi:10.1186/ 1472-6939-9-15.

Morrison, J., M. Pai, and P.C. Hopewell. 2008. Tuberculosis and latent tuberculosis infection in close contacts of people with pulmonary tuberculosis in low-income and middle-income countries: A systematic review and meta-analysis. The Lancet Infectious Diseases 8(6): 359-368. doi:10.1016/ S1473-3099(08)70071-9.

National Institute for Health and Clinical Excellence (NICE). 2006. Tuberculosis: Clinical diagnosis and management of tuberculosis, and measures for its prevention and control. http://www.nice.org.uk/nicemedia/pdf/CG033niceguide line.pdf. Accessed 4 April 2009.
Orme, I.M. 2005. The use of animal models to guide rational vaccine design. Microbes and Infection 7(5-6): 905-910. doi:10.1016/j.micinf.2005.03.008.

Samson, K. 2008. Orphan economics: The downside of supplyside pharmacology. Annals of Neurology 64(3): A13-A16. doi:10.1002/ana.21432.

Selgelid, M.J. 2005. Ethics and infectious disease. Bioethics 19 (3): 272-289. doi:10.1111/j.1467-8519.2005.00441.x.

Selgelid, M.J., P.M. Kelly, and A. Sleigh. 2008a. Ethical challenges in $\mathrm{TB}$ control in the era of XDR-TB. The International Journal of Tuberculosis and Lung Disease 12(3): 231-235.

Selgelid, M.J., P.M. Kelly, and A. Sleigh. 2008b. In International Public Health Policy and Ethics, ed. M. Boylan. Dordrecht: Springer.

Shisana, O., E.J. Hall, R. Maluleke, J. Chauveau, and C. Schwabe. 2004. HIV/AIDS prevalence among South African health workers. South African Medical Journal 94(10): 846-850.

Snider Jr., D.E. 2000. Ethical issues in tuberculosis vaccine trials. Clinical Infectious Diseases 30(Suppl 3): S271S275. doi:10.1086/313872.

Soliman, E.Z. 2008. The ethics of non-inferiority trials. Lancet 371(9616): 895. doi:10.1016/S0140-6736(08)60406-7. author reply 896-7.

Tascon, R.E., M.J. Colston, S. Ragno, E. Stavropoulos, D. Gregory, and D.B. Lowrie. 1996. Vaccination against tuberculosis by DNA injection. Nature Medicine 2(8): 888-892. doi:10.1038/nm0896-888.

U.S. Department of Health, Education and Welfare. (1979). The Belmont report: Ethical principles and guidelines for the protection of human subjects of research. Federal Register, 44(76): 23191-23197. http://ohsr.od.nih.gov/guidelines/ belmont.html. Accessed 4 April 2009.

Williams, A., G.J. Hatch, S.O. Clark, K.E. Gooch, K.A. Hatch, G.A. Hall, K. Huygen, T.H. Ottenhoff, K.L. Franken, P. Andersen, T.M. Doherty, S.H. Kaufmann, L. Grode, P. Seiler, C. Martin, B. Gicquel, S.T. Cole, P. Brodin, A.S. Pym, W. Dalemans, J. Cohen, Y. Lobet, N. Goonetilleke, H. McShane, A. Hill, T. Parish, D. Smith, N.G. Stoker, D. B. Lowrie, G. Kallenius, S. Svenson, A. Pawlowski, K. Blake, and P.D. Marsh. 2005. Evaluation of vaccines in the EU TB Vaccine Cluster using a guinea pig aerosol infection model of tuberculosis. Tuberculosis (Edinburgh, Scotland) 85(1-2): 29-38. doi:10.1016/j.tube.2004.09.009.

World Health Organization. 2007. Global tuberculosis control: surveillance, planning, financing. WHO Report. Geneva: World Health Organisation.

World Medical Association. 2008. Declaration of Helsinki. http:// www.wma.net/e/policy/b3.htm. Accessed 4 April 2009. 